\title{
Cholesterol hydroxylation products confer resistance to anthracyclines in Triple Negative Breast Cancer
}

\author{
S.A. Hutchinson ${ }^{1}$, D.A. Kane ${ }^{1}$, H. Røberg-Larsen ${ }^{2}$, K. Vaghela ${ }^{1}$ and J.L. Thorne ${ }^{1}$ \\ ${ }^{1}$ School of Food Science and Nutrition, University of Leeds, LS2 9JT and \\ ${ }^{2}$ Department of Chemistry, University of Oslo, Norway and School of Medicine.
}

For Triple Negative Breast Cancer (TNBC) patients, treatment commonly consists of surgery, local radiotherapy and systemic chemotherapy. Prognosis following metastatic relapse (which indicates failure of systemic chemotherapy) is poor as fewer than 1 in 10 of these patients survive longer than two-years. The 2017 WCRF Breast Cancer Continuous Update Project ${ }^{(1)}$ reported convincing risk factors for $\mathrm{BCa}$ that are common comorbidities with hypercholesterolemia (e.g. obesity, waist-hip-ratio). At the nutrient-gene interaction level this is intriguing because cholesterol is hydroxylated into oxysterols (OHCs), a group of potent signaling molecules that drive TNBC metastasis through the Liver X Receptor (LXR) $)^{(2)}$ and are elevated in serum at metastatic relapse ${ }^{(3)}$. Dietary ${ }^{(4)}$ and pharmacological $^{(5)}$ interventions that lower circulating concentrations of cholesterol and oxysterols, also improve BCa disease free survival. Dietary phytosterols in particular not only reduce circulating cholesterol and OHC concentrations, but their structural similarity to OHCs suggests they are modulators of the LXR-OHC axis. The aims of this study were to evaluate $i$ ) if the LXR-OHC axis confers chemoresistance in $\mathrm{BCa}$, and $i$ i) if phytosterols antagonise this pathway.

Pretreatment of TNBC cells in culture with LXR specific ligands led to altered intra-cellular retention of the fluorescent anthracycline, epirubicin (Fig 1) and altered epirubicin induced cell death in colony forming assays (Fig 2). To test if phytosterols could impair the oxysterol-LXR axis, stable LXR-reporter cell lines were generated. Transactivation of LXR was inhibited in the presence of Sitosterol or the synthetic LXR antagonist GSK2033 (Fig 3). Sitosterol treatment was also found to significantly impair TNBC mammosphere formation (Fig 4).
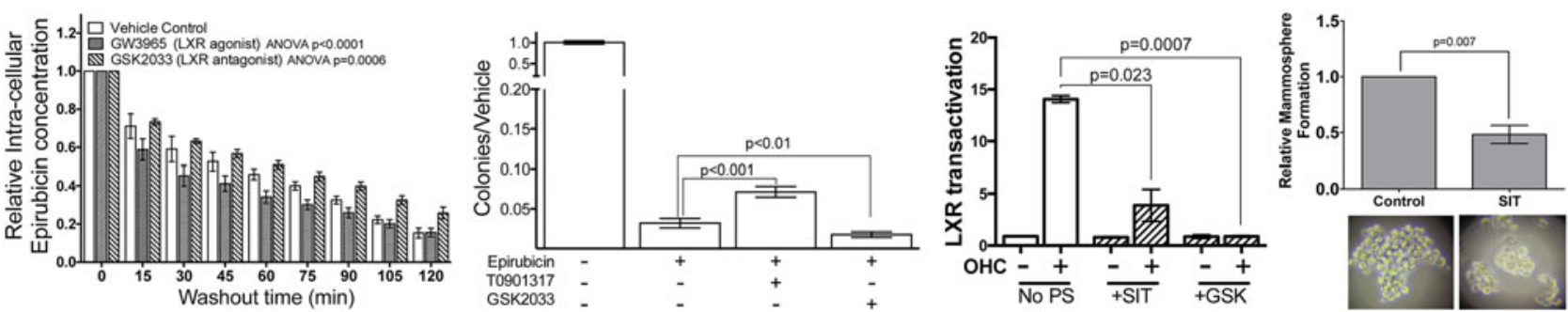

Fig. 1. Modulating LXR activity with agonist or antagonist enhances the cell's ability to efflux epirubicin (100uM). Fig. 2. Colony Forming Assay show LXR activity modifies cellular survival in response to epirubucin. Fig. 3. Transactivation of LXR by OHC is impaired by synthetic antagonist and Sitosterol (SIT). Fig. 4. Mammopshere formation is impaired by Sitosterol. All data show mean of 2-5 independent biological replicates. Student's t-test or ANOVA were used to assess statistical significance.

Targeting LXR transcriptional capacity with highly specific ligands led to changes in retention of epirubicin and tumour cell survival. Phytosterol treatment impaired the ability of an endogenous OHC to transactivate LXR and for TNBC cells to form mammospheres. We have now begun measuring $\mathrm{OHC}$ and phytosterol content of primary tumours to evaluate if intra-tumoural concentrations of OHCs are sufficient to transactivate LXR and if they are functional biomarkers of tumour relapse. Giventhe ease with which phytosteorls could be adopted into therapeutic nutritional advice we propose they should be evaluated as modifiers of the chemoresponse in TNBC patients, particularly those who present with elevated circulating cholesterol.

1 WCRF/AICR. Continuous Update Project Report: Diet, Nutrition, Physical Actvity and Cancer 2017.

2 Nelson ER et al. (2013) Science 342, 1094-8.

3 Dalenc F et al. (2016) J Steroid Biochem Mol Biol 169, 210-8.

4 Chlebowski RT et al. (2006) J Natl Cancer Inst 98, 1767-76.

5 Liu B, Yi Z, Guan X, Zeng YX \& Ma F (2017) Breast Cancer Res Treat 164, 1-11. 